\title{
Study on the definition of equivalent plastic strain under non-associated flow rule for finite element formulation
}

\author{
Mohsen Safaei ${ }^{\mathrm{a}}$, Jeong Whan Yoon ${ }^{\mathrm{b}, *}$, Wim De Waele ${ }^{\mathrm{a}}$ \\ a Department of Mechanical Construction and Production, Faculty of Engineering and Architecture, Ghent University, Technologiepark Zwijnaarde 903, \\ Zwijnaarde BE9052, Belgium \\ ${ }^{\mathrm{b}}$ School of Engineering, Deakin University, Geelong Waurn Ponds, VIC 3220, Australia
}

\section{A R T I C L E I N F O}

\section{Article history:}

Received 9 July 2013

Received in final revised form 26 September 2013

Available online 8 October 2013

\section{Keywords:}

Non associated flow rule

Anisotropy

Equivalent plastic strain

Cup deep drawing

Plastic potential

\begin{abstract}
A B S T R A C T
As opposed to associated flow rule (AFR) in which yield function and plastic potential are equal, the different definitions for them is an inherent characteristic of non-associated flow rule (non-AFR). This imposes a specific relation (but not equality) between equivalent plastic strain and plastic compliance factor. Unavoidably, this leads to a laborious effort for FE implementation of non-associated constitutive model specifically when several internal variables (such as kinematic hardening or damage parameters) are involved. This paper is mainly devoted to studying the conditions at which the non-AFR approach can be simplified so that the numerical implementation scheme is more convenient without loss of accuracy. It will be shown that by scaling the plastic potential function, the equality of equivalent plastic strain and compliance factor can be reserved. The effect of scaling of the non-AFR based on Barlat et al.'s (2003) anisotropic model (called Yld2000-2d) is comprehensively studied with FE simulation of tensile loading under uniaxial tensions along the different orientations as well as balanced biaxial stress condition. A fully implicit return-mapping scheme was introduced for stress integration of the constitutive model in a User-defined MATerial subroutine (UMAT). Cup drawing simulations of a highly textured aluminum alloy 2090-T3 were performed using simplified and original approaches. The results prove that the proposed simplified technique is a reliable alternative for the full expression.
\end{abstract}

(c) 2013 Elsevier Ltd. All rights reserved.

\section{Introduction}

Various phenomenological yield functions have been proposed to simulate the anisotropic behaviors of metals. Most anisotropic yield functions are based on the associated flow rule (AFR) hypothesis obeying the normality rule. Accordingly, under the assumption of AFR with the light of material orthotropy, various phenomenological yield functions have been proposed to describe the initial anisotropy of metallic sheets including Hill's (1948), Barlat et al. (1991, 2003, 2005, 2007), Karafillis and Boyce (1993), Cazacu and Barlat (2002, 2004), Bron and Besson (2004), Cazacu et al. (2004, 2006), Vegter and van den Boogaard (2006), Hu (2007), etc. Barlat et al. (2011) proposed an alternative approach to consider kinematic hardening within the framework of anisotropic yield function under associated flow rule. Recently, Cleja-Tigoiu and Iancu (2013) considered an orthotropic non-quadratic yield function dependent on the third invariant of the stress and showed that the plastic spin provides the change in the orthotropy axes, characterized by the Euler angles.

\footnotetext{
* Corresponding author. Tel.: +61 352273425 .

E-mail address: j.yoon@deakin.edu.au (J.W. Yoon).
} 
During the last decade, some attention has been paid on the development and implementation of non-AFR for metal plasticity. Stoughton (2002) proposed a non-AFR model based on Hill (1948) quadratic formulation that accurately predicted both direction dependent $r$-values and yield stresses in rolling, transverse and diagonal directions. Continuing his previous model, Stoughton and Yoon (2004) developed a pressure sensitive non-AFR model that predicted the strength differential effect observed in tension and compression tests. Stoughton and Yoon $(2006,2008)$ derived the stability conditions for non-associated flow plasticity. Cvitanic et al. (2008) developed a non-AFR model based on both Hill (1948) and Karafillis and Boyce non-quadratic yield functions combined with isotropic hardening, which demonstrated improved height predictions for deep drawn cups. Stoughton and Yoon (2009) proposed a non-AFR based anisotropic hardening model that resulted in excellent predictions of hardening curves for rolling, transverse and diagonal directions and for the balanced biaxial stress state. Improvements in prediction of cup height and springback of a U-bend specimen using non-AFR with mixed isotropic-kinematic hardening have been also reported by Taherizadeh et al. (2010, 2011). Gao et al. (2011) showed the significance of the hydrostatic stress on plastic response with the non-associated flow rule. Park and Chung (2012) derived a symmetric stiffness modulus for the non-associated flow rule under the framework of the combined isotropic-kinematic hardening law. Recently, Safaei et al. (2013a) proposed an evolutionary anisotropic model based on non-AFR that excellently predicted distortional hardening and evolution of instantaneous $r$-values in seven uniaxial directions as well as balanced biaxial loading condition.

Among many benefits of using non-associated flow for anisotropic plasticity, non-AFR hypothesis removes the constraint of the normality rule where plastic potential and yield function are equal under associated flow rule. Consequently, two separate functions for yield and plastic potential can be adopted. In other words, the yield function and plastic potential describe the elastic limit and plastic strain rate direction independently. Then, the hardening of yield function and the direction of plastic flow can be separated resulting in the uncoupled predictions of stress ratios and $r$-values.

Considering the increasing popularity of non-AFR in metal forming simulations, this paper is aimed to propose a simple implementation based on fully implicit integration scheme. The motivation is that the combination of a non-AFR model with complex hardening model or damage parameters is a cumbersome task due to inequality of plastic compliance factor and equivalent plastic strain. Consequently, this paper proposes a method to reduce the degree of inaccuracy with simplification. First, we briefly discuss the development of a non-AFR Yld2000-2d (Barlat et al., 2003). Then, the stress and Lankford directionalities predicted from AFR and non-AFR Yld2000-2d are compared for two highly anisotropic materials such as an interstitial free steel DC06 and AA2090-T3. The developed (simplified and full) models were implemented into a commercial FE code ABAQUS using a fully implicit return mapping algorithm (backward Euler method). The multi-stage return mapping method based on the incremental deformation theory proposed by Yoon et al. (1999) is used in the implementation to enhance the convergence of the linearization algorithm for large strain increments. Subsequently, comprehensive comparisons are provided for the simplified and full methods. Finally, the results of cup deep drawing simulations based on the evaluated models are discussed.

\section{Review of associated flow rule (AFR)}

Associated flow rule (AFR) reflects the normality rule that describes that the gradient of the yield surface determines the direction of plastic flow. We can write a yield criterion under AFR as

$$
F=f_{y}(\underset{\sim}{\sigma})-\sigma^{i s o}\left(\bar{\varepsilon}^{p}\right)
$$

where $f_{y}$ and $\sigma^{\text {iso }}$ respectively denote yield function and isotropic hardening; $\bar{\varepsilon}^{p}$ is equivalent plastic strain. The normality rule is given by

$$
d \varepsilon_{\sim}^{p}=d \lambda \underset{\sim}{m}
$$

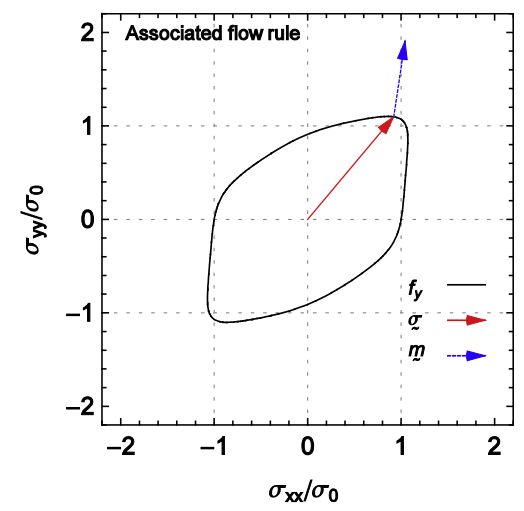

Fig. 1. Concept of normality rule ( $f_{y}$ is yield function; $\sigma$ is Cauchy stress; $m$ is plastic strain rate direction). 
where

$$
\underset{\sim}{m}=\partial f_{y} / \partial \underset{\sim}{\sigma}
$$

$d \lambda$ is the plastic multiplier factor (compliance) to be determined under plastic deformation, and the second order tensor $m$ is the plastic flow direction (Simo and Hughes, 1998). According to Bishop and Hill (1951), the normality hypothesis is theoretically valid for polycrystals. In addition, Hecker (1976) described that the normality hypothesis is reasonable for most single phase materials. The normality rule is illustrated in Fig. 1 in the normalized stress space.

\section{Non-associated flow rule (non-AFR)}

\subsection{Review of non-AFR}

The residual $F$ in non-AFR is similar to the one in AFR shown in Eq. (1). However, as opposed to Eq. (2), the plastic flow direction under non-AFR approach can be determined by a plastic potential. Accordingly, non-associated plastic flow rule is described by

$$
d \varepsilon_{\sim}^{p}=d \lambda \underset{\sim}{\sim}
$$

where

$$
\underset{\sim}{n}=\partial f_{p} / \partial \underset{\sim}{\sigma}
$$

$f_{p}$ is a plastic potential and the second order tensor $n$ is the plastic flow direction i.e. normal to the plastic potential. It is noticed that due to the use of plastic potential $f_{p}$ instead of yield function $f_{y}$, the normality hypothesis is no longer valid in non-AFR. Non-AFR for AA2090-T3 is shown in Fig. 2 as an example.

\subsection{Non-associated Yld2000-2d}

A non-quadratic plane stress yield model proposed by Barlat et al. (2003) (called Yld2000-2d) gained considerable popularity mainly because of its accurate prediction of yield stress and $r$-value directionalities covering rolling, diagonal, and transverse directions as well as balanced biaxial stress state. This yield function is based on a linear transformation of two unconditionally convex functions $\varphi^{\prime}$ and $\varphi^{\prime \prime}$ of deviatoric stress tensor:

$$
f_{y}=\left[\frac{1}{2}\left(\varphi^{\prime}+\varphi^{\prime \prime}\right)\right]^{\frac{1}{m}}
$$

where

$$
\varphi^{\prime}=\left(X_{1}^{\prime}-X_{2}^{\prime}\right)^{m}, \quad \varphi^{\prime \prime}=\left(2 X_{2}^{\prime \prime}+X_{1}^{\prime \prime}\right)^{m}+\left(2 X_{1}^{\prime \prime}+X_{2}^{\prime \prime}\right)^{m}
$$

The exponent $m$ is associated to crystallographic structure and is recommended to be 6 for BCC and 8 for FCC type metals. $X_{1,2}^{\prime}$ and $X_{1,2}^{\prime \prime}$ are the principal values of the linear transformation of the stress deviators $\tilde{\mathbf{s}}^{\prime}$ and $\tilde{\mathbf{s}}^{\prime \prime}$ respectively.

$$
\tilde{\mathbf{s}}^{\prime}=L^{\prime} . \boldsymbol{\sigma}, \quad \tilde{\mathbf{s}}^{\prime \prime}=L^{\prime \prime} . \boldsymbol{\sigma}
$$

with

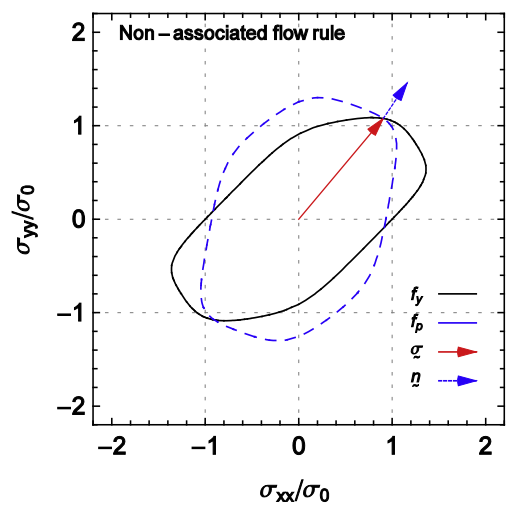

Fig. 2. Concept of the non-associated flow rule ( $f_{y}$ is yield function; $f_{p}$ is plastic potential function; $\sigma$ is Cauchy stress; $n$ is plastic strain rate direction). 


$$
\left[\begin{array}{l}
L_{11}^{\prime} \\
L_{12}^{\prime} \\
L_{21}^{\prime} \\
L_{22}^{\prime} \\
L_{66}^{\prime}
\end{array}\right]=\left[\begin{array}{ccc}
2 / 3 & 0 & 0 \\
-1 / 3 & 0 & 0 \\
0 & -1 / 3 & 0 \\
0 & 2 / 3 & 0 \\
0 & 0 & 1
\end{array}\right]\left[\begin{array}{l}
\alpha_{1} \\
\alpha_{2} \\
\alpha_{7}
\end{array}\right]
$$

$$
\left[\begin{array}{l}
L_{11}^{\prime \prime} \\
L_{12}^{\prime \prime} \\
L_{21}^{\prime \prime} \\
L_{22}^{\prime \prime} \\
L_{66}^{\prime \prime}
\end{array}\right]=\frac{1}{9}\left[\begin{array}{ccccc}
-2 & 2 & 8 & -2 & 0 \\
1 & -4 & -4 & 4 & 0 \\
4 & -4 & -4 & 1 & 0 \\
-2 & 8 & 2 & -2 & 0 \\
0 & 0 & 1 & 0 & 9
\end{array}\right]\left[\begin{array}{l}
\alpha_{3} \\
\alpha_{4} \\
\alpha_{5} \\
\alpha_{6} \\
\alpha_{8}
\end{array}\right]
$$

The principal values of the transformed stress deviator $\tilde{\mathbf{s}}^{\prime}$ denoted by $X_{1}^{\prime}$ and $X_{2}^{\prime}$ can be calculated as

$$
\begin{aligned}
& X_{1}^{\prime}=\left(\tilde{s}_{11}^{\prime}+\tilde{S}_{22}^{\prime}\right) / 2+\sqrt{\tilde{s}_{12}^{\prime}{ }^{2}+\left(\left(\tilde{S}_{11}^{\prime}-\tilde{S}_{22}^{\prime}\right) / 2\right)^{2}} \\
& X_{2}^{\prime}=\left(\tilde{s}_{11}^{\prime}+\tilde{S}_{22}^{\prime}\right) / 2-\sqrt{\tilde{s}_{12}^{\prime}{ }^{2}+\left(\left(\tilde{S}_{11}^{\prime}-\tilde{S}_{22}^{\prime}\right) / 2\right)^{2}}
\end{aligned}
$$

Similar formulations apply for $X_{1}^{\prime \prime}$ and $X_{2}^{\prime \prime}$.

Considering AFR Yld2000-2d, eight experimental results such as yield stress and r-value for rolling, transverse and diagonal directions and for balanced biaxial stress state $\left(r_{0}, r_{45}, r_{90}, r_{b}, \sigma_{0}, \sigma_{45}, \sigma_{90}, \sigma_{b}\right)$, are required to determine the model parameters. On the other hand, in non-AFR Yld2000-2d, due to the flexibility to separate stress-ratios and r-values with two functions, the parameters of yield function are optimized solely based on directional yield stresses at every $15^{\circ}$ from rolling direction $\left(\sigma_{0}, \sigma_{15}, \sigma_{30}, \sigma_{45}, \sigma_{60}, \sigma_{75}, \sigma_{90}\right)$ as well as that of balanced biaxial stress $\left(\sigma_{b}\right)$. Furthermore, parameters of the plastic potential can incorporate Lankford coefficients for unidirectional loading in different orientations $\left(r_{0}, r_{15}, r_{30}, r_{45}, r_{60}, r_{75}, r_{90}\right)$ as well as that of balanced biaxial loading $\left(r_{b}\right)$. So, 8 parameters under AFR increase to 16 parameters when non-AFR is used, which is the same number of plane stress parameters for Barlat et al.'s (2005) yield function under AFR. This methodology was presented by Yoon et al. (2007) and recently by Park and Chung (2012).

\section{Prediction of anisotropy}

The interstitial free deep drawing grade steel DC06 and AA2090-T3 were selected for the comparison of AFR and non-AFR Yl2000-2d models. The texture of a DC06 sheet was determined by means of pole figure measurements by X-ray diffraction and its subsequent calculation of the orientation distribution function (ODF) using the MTM-FHM software (Van Houtte, 1995). The $r$-values and normalized yield stresses are obtained from the ODF using the VEF software with polycrystal plasticity calculations and assuming the full constraints (FC) Taylor theory with $\{110\}+\{112\}\langle 110\rangle$ crystallographic slip (Van Houtte et al., 2005, 2011; Gawad et al., 2013). The normalized yield stresses and $r$-values (either experimentally (AA 2090-T3) determined or obtained from Taylor method (DC06)) are presented in Tables 1 and 2, respectively. The experimental data for AA2090-T3 are given in (Chung et al., 1996; for details at Yoon et al., 2000). Parameter identification was performed using an inverse approach and the technique presented in the work of Yoon et al. (2004). The calculated parameters for AA2090-T3 and DC06 are given in Table 3.

Figs. 3 and 4 show both yield function and plastic potential respectively for DC06 and AA2090-T3 predicted from AFR and non-AFR with Yld2000-2d. Figs. 5 and 6 present the predicted normalized yield stress and Lankford coefficient directionalities compared to either experimentally (AA2090-T3) determined values or the values obtained through Taylor method (DC06). It can be seen that the AFR Yld-2000-2d model captures $0^{\circ}, 45^{\circ}$ and $90^{\circ}$ orientations. However, the dominant accuracy of non-AFR approach over its AFR counterpart is clear from these plots due to the flexibility to incorporate more parameters from the separation of yield function and plastic potential.

Table 1

Normalized yield stresses.

\begin{tabular}{lllllllll}
\hline & $0^{\circ}$ & $15^{\circ}$ & $30^{\circ}$ & $45^{\circ}$ & $60^{\circ}$ & $75^{\circ}$ & $90^{\circ}$ & $b$ \\
\hline AA2090-T3 (Exp.) & 1.000 & 0.961 & 0.910 & 0.811 & 0.810 & 0.882 & 0.910 \\
DC06 (Taylor) & 1.000 & 1.006 & 1.013 & 1.009 & 1.005 & 1.007 & 1.012 & 1.035 \\
\hline
\end{tabular}


Table 2

Lankford coefficients.

\begin{tabular}{|c|c|c|c|c|c|c|c|c|}
\hline & $0^{\circ}$ & $15^{\circ}$ & $30^{\circ}$ & $45^{\circ}$ & $60^{\circ}$ & $75^{\circ}$ & $90^{\circ}$ & $b$ \\
\hline AA2090-T3 (Ехр.) & 0.212 & 0.327 & 0.692 & 1.577 & 1.039 & 0.538 & 0.692 & 0.670 \\
\hline DC06 (Taylor) & 2.204 & 2.278 & 2.303 & 2.127 & 2.127 & 2.372 & 2.492 & 0.910 \\
\hline
\end{tabular}

Table 3

Yld2000-2d (AFR and non-AFR) model parameters.

\begin{tabular}{|c|c|c|c|c|c|c|c|c|c|}
\hline & $\alpha_{1}$ & $\alpha_{2}$ & $\alpha_{3}$ & $\alpha_{4}$ & $\alpha_{5}$ & $\alpha_{6}$ & $\alpha_{7}$ & $\alpha_{8}$ & $m$ \\
\hline \multicolumn{10}{|l|}{ АА2090-T3 } \\
\hline Potential $\left(\mathrm{f}_{\mathrm{p}}\right)$ & -0.856 & 1.154 & -0.293 & 0.326 & 0.683 & 0.482 & 0.752 & 1.024 & 8 \\
\hline Yield $\left(\mathrm{f}_{\mathrm{y}}\right)$ & -0.713 & 2.037 & 1.629 & 0.69 & 0.552 & -1.057 & 1.255 & 1.263 & 8 \\
\hline AFR & 0.488 & 1.377 & 0.754 & 1.025 & 1.036 & 0.904 & 1.231 & 1.485 & 8 \\
\hline \multicolumn{10}{|l|}{ DC06 } \\
\hline Potential $\left(f_{p}\right)$ & 0.811 & 1.827 & 2.755 & 1.336 & -0.831 & 0.227 & 1.303 & 0.800 & 6 \\
\hline Yield $\left(f_{y}\right)$ & -1.256 & 1.705 & -0.693 & 0.648 & 0.983 & 0.727 & -0.311 & 1.625 & 6 \\
\hline AFR & 1.068 & 1.041 & 0.983 & 0.918 & 0.935 & 0.995 & 1.046 & 0.881 & 6 \\
\hline
\end{tabular}
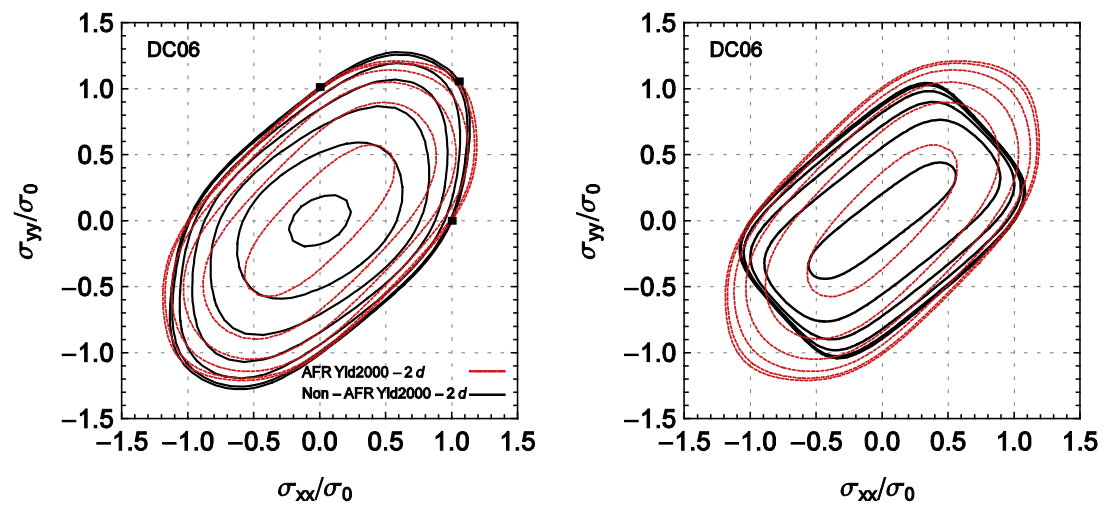

Fig. 3. DC06: Normalized yield function (left) and normalized plastic potential with Yld2000-2d model (right).
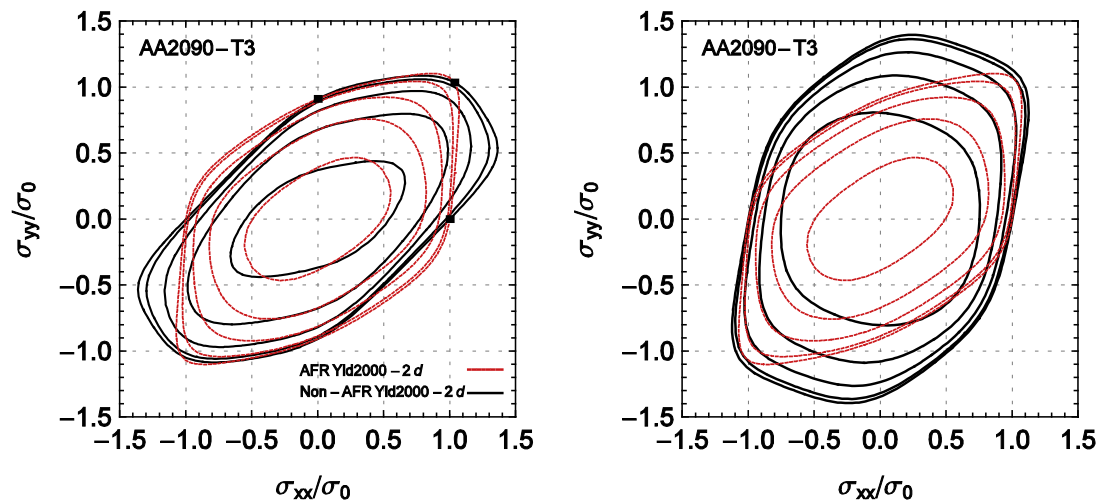

Fig. 4. AA2090-T3: Normalized yield function (left) and normalized plastic potential with Yld2000-2d model (right)

\section{Equivalent plastic strain in a non-AFR model}

5.1. Relation between equivalent plastic strain and plastic multiplier factor

A scaled (simplified) non-AFR model is investigated in this section. The aim is to alleviate the laborious task of a fully implicit backward Euler method. 


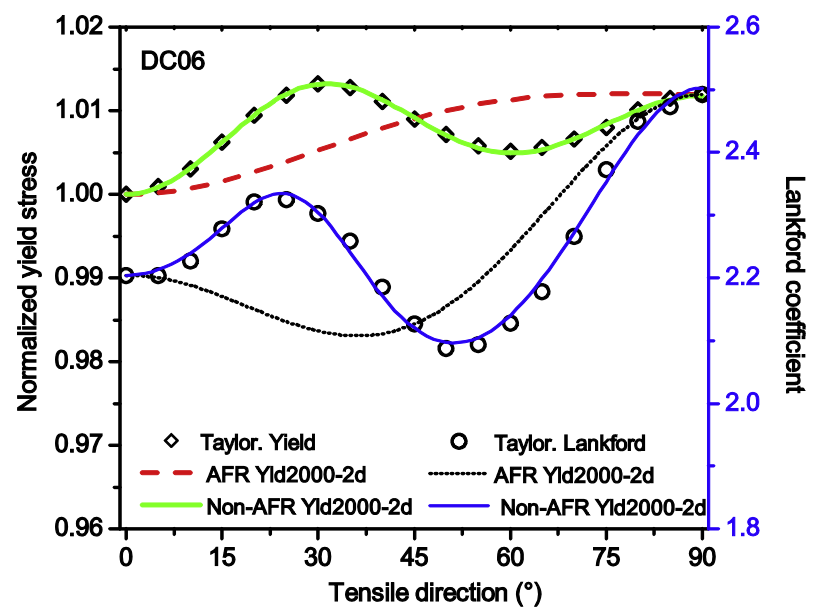

Fig. 5. Normalized tensile yield stress and Lankford coefficient distribution for DC06.

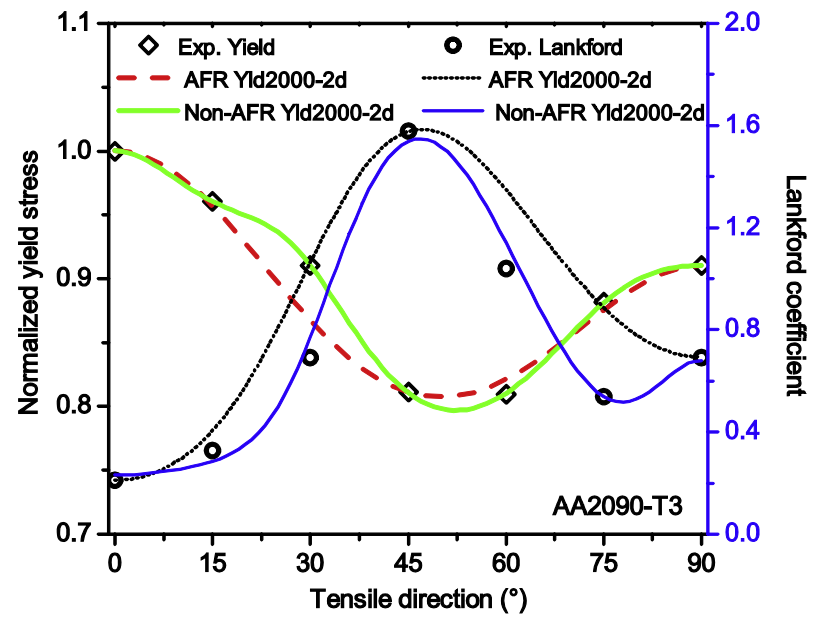

Fig. 6. Normalized tensile yield stress and Lankford coefficient distribution for AA2090-T3.

The principle of plastic work equivalence yields the following relation between equivalent plastic strain and plastic strain tensor

$$
f_{y} d \bar{\epsilon}^{p}=\underset{\sim}{\sigma}: d \varepsilon_{\sim}^{p}
$$

where $f_{y}$ is a yield function. Euler's theorem for any first order homogeneous function $f$ states

$$
\underset{\sim}{\sigma}: \frac{\partial f}{\partial \bar{\sigma}}=f
$$

Applying this on $f_{y}$ or $f_{p}$ results in

$$
\begin{aligned}
& \underset{\sim}{\sigma}: \underset{\sim}{n}=f_{p} \\
& \sigma: m=f_{y}
\end{aligned}
$$

Substituting Eq. (4) into Eq. (11) and applying Euler's theorem in Eq. (13) results in

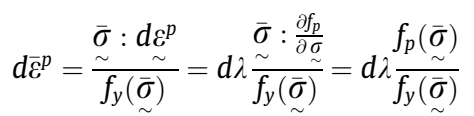

Eq. (14) can be reduced to the following equation for a special case (to be discussed in Section 6.3) 


$$
d \bar{\varepsilon}^{p}=d \lambda
$$

Eq. (15) has been adopted by Stoughton (2002), Stoughton and Yoon (2006, 2008, 2009), Taherizadeh et al. (2010) and Safaei et al. (2013b) by simply making equal a uniaxial tension behavior along the rolling for yield function and plastic potential (called "scaled simplified approach" in this paper). This is because it results in a simpler implementation of the non-AFR constitutive model using a fully implicit backward Euler scheme. The detailed verification for Eq. (15) (for scaled simplified case) has been conducted in this paper by comparing the results from Eq. (14) and un-scaled simplified case.

\subsection{Fully implicit integration scheme}

As a fully implicit integration scheme has been adopted, increments in the plastic strain and flow direction are attributed to the current step denoted by $n+1$. The following integration scheme is written for the non-AFR model with isotropic hardening and a general anisotropic yield function.

$$
\begin{aligned}
& \underset{\sim}{\varepsilon_{(n+1)}}={\underset{\sim}{\varepsilon}}_{(n)}+\Delta \underset{\sim}{\varepsilon} \\
& \varepsilon_{\sim}^{p}{ }_{(n+1)}=\varepsilon_{\sim}^{p} p_{(n)}+\Delta \lambda_{(n+1)} n_{\sim} n_{(n+1)} \\
& \bar{\varepsilon}_{(n+1)}^{p}=\bar{\varepsilon}_{(n)}^{p}+\Delta \lambda_{(n+1)}\left(\frac{f_{p}}{f_{y}}\right)_{(n+1)}
\end{aligned}
$$

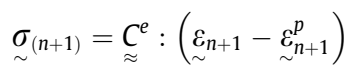

$$
\begin{aligned}
& F_{n+1}\left(\sigma_{(n+1)}, \bar{\varepsilon}_{(n+1)}^{p}\right)=0
\end{aligned}
$$

This set of equations has to be linearized with respect to $\bar{\varepsilon}^{p}$ at the time increment $\Delta t_{n+1}=t_{n+1}-t_{n}$. The difference between the right and left sides from Eq. (16a)-(16e) defines the corresponding residual functions. The values of these residuals are minimized within a very small tolerance by means of Newton-Raphson iteration scheme. More specifically, to include the full expression of Eq. (16c) in a fully implicit integration scheme a residual function $r^{p}$ is defined

$$
r^{p}=\Delta \bar{\varepsilon}_{(n+1)}^{p} f_{y,(n+1)}-\Delta \lambda_{(n+1)} f_{p,(n+1)}
$$

Constructing the truncated Taylor expansion of $r^{p}$ leads to

$$
r^{p(\text { taylor })}=r^{p}+\dot{\bar{\varepsilon}}_{k}^{p} f_{y}+\Delta \bar{\varepsilon}_{k}^{p} \dot{f}_{y}-\dot{\lambda}_{k} f_{p}-\Delta \lambda_{k} \dot{f}_{p}
$$

The superposed dot denotes the incremental change during the kth Newton-Raphson iteration. Using Euler's rule

$$
\begin{aligned}
& \dot{f}_{p}=\underset{\sim}{n}: \underset{\sim}{\dot{\sigma}} \\
& \dot{f}_{y}=\underset{\sim}{m}: \dot{\sim}
\end{aligned}
$$

Alternatively, if the simplified expression is used, then Eq. (16c) can be substituted by a linear function in which the derivative of $f_{p} / f_{y}$ can be eliminated

$$
\bar{\varepsilon}_{(n+1)}^{p}=\bar{\varepsilon}_{(n)}^{p}+\Delta \lambda_{(n+1)}
$$

Apparently, Eqs. (17) and (18) are omitted if the simplification is used.

Regarding the details of the integration scheme, Cvitanic et al. (2008) presented a fully implicit scheme for a non-associated flow rule in the case of isotropic hardening based on multi-step return mapping method by Yoon et al. (1999). Taherizadeh et al. (2010) and Safaei et al. (2013b) developed a fully implicit scheme for different mixed isotropickinematic hardening models. Due to the fact, only isotropic hardening is considered in this paper thus integration scheme is similar to that of Cvitanic et al. (2008) so the reader is referred to that paper for further details.

\section{Impact of simplification on equivalent plastic strain rate}

For the sake of convenience, the following terminology is agreed upon

$$
d \bar{\varepsilon}^{p}=d \lambda \frac{f_{p}}{f_{y}} \text { in Eq. (14) } \rightarrow \text { full method }
$$

$d \bar{\varepsilon}^{p}=d \lambda$ in Eq. (15) with the original $f_{p} \rightarrow$ un-scaled simplified method

$d \bar{\varepsilon}^{p}=d \lambda$ in Eq. (15) with the scaled $f_{p} \rightarrow$ scaled simplified method

Based on these approaches, the non-AFR constitutive model is evaluated and results are presented in the following subsections. 


\subsection{Full non-AFR method}

From Eq. (14) one obtains

$$
d \lambda=d \bar{\varepsilon}^{p} \frac{f_{y}}{f_{p}}
$$

If $d \lambda$ in Eq. (21) is substituted into the non-associated flow rule in Eq. (4), the following equation is obtained

$$
d \varepsilon_{\sim}^{p}=d \bar{\varepsilon}^{p} \frac{f_{y}}{f_{p}} \underset{\sim}{n}
$$

It would be advantageous to consider all the equations in normalized stress space; normalized with respect to the yield stress in the rolling direction denoted by $\sigma_{0}$. In the remainder of this section, the notation $\sigma$ refers to the normalized stress. Therefore the normalized uniaxial stress state with only a non-zero stress component in rolling direction is represented by

$$
\begin{aligned}
\sigma_{x x} & =1 \\
\sigma_{y y} & =0 \\
\sigma_{x y} & =0
\end{aligned}
$$

Substitution of Eq. (23) into Euler's theorem Eq. (13a) leads to

$$
f_{p}=n_{x x}
$$

In normalized stress space, yielding occurs when

$$
f_{y}=1
$$

Substitution of Eq. (23) and Eq. (25) into Euler's theorem Eq. (13b), leads to

$$
f_{y}=m_{x x}=1
$$

If Eqs. (24) and (26) are substituted into Eq. (22) for an uniaxial stress state, the following equation is obtained

$$
d \varepsilon_{x x}^{p}=d \bar{\varepsilon}^{p}
$$

In short, the full expression automatically equalizes the longitudinal plastic strain in the rolling direction $\left(\varepsilon_{x x}^{p}\right)$ with the equivalent plastic strain $\left(\bar{\varepsilon}^{p}\right)$.

\subsection{Un-scaled simplified non-AFR method}

The un-scaled simplified model is based on elimination of the term $f_{p} / f_{y}$ in Eq. (16c) with no additional stipulations. Substitution of $d \bar{\varepsilon}^{p}=d \lambda$ into the non-associated flow rule in Eq. (4) leads to

$$
\left(d \varepsilon_{\sim}^{p}=d \bar{\varepsilon}^{p} n_{\sim}^{\text {un-scaled simplified }}\right.
$$

or alternatively

$$
\left(d \bar{\varepsilon}^{p}=d \varepsilon^{p} / n\right)^{\text {un-scaled simplified }}
$$

From Euler's theorem in Eq. (13a) and in case $f_{p} \neq 1$ when yielding occurs, the first component of for uniaxial stress along the rolling direction does not equal 1

$$
n_{x x} \neq 1
$$

Consequently, combining Eq. (29) with Eq. (28a) for uniaxial stress state along the rolling direction, leads to

$$
d \varepsilon_{x x}^{p} \neq d \bar{\varepsilon}^{p}
$$

Therefore, if no constraint is enforced to the un-scaled simplified approach, this method results in a wrong relation between equivalent plastic strain and longitudinal plastic strain along the rolling direction.

Considering a general stress state, determination of the ratio of equivalent plastic strain (obtained from the full method) to equivalent plastic strain (obtained from the un-scaled method), $\beta^{\text {un-scaled simplified }}$, can be calculated as the ratio of Eq. (22) to Eq. (28a). After some manipulations

$$
\frac{\left.d \bar{\varepsilon}^{p}\right|^{\text {full method }}}{\left.d \bar{\varepsilon}^{p}\right|^{\text {un-scaled simplified }}}=\frac{f_{p}}{f_{y}}=\beta^{\text {un-scaled simplified }}
$$

Fig. 7 plots the variation of parameter $\beta^{\text {un-scaled simplified }}$ for different stress states at the first quarter of the Yld2000-2d yield surface for AA2090-T3. A cylindrical coordinate system is introduced, Fig. 8, to enable a straightforward evaluation of the 
$\sigma_{x x}=1, \sigma_{y y}=0, \beta=1$

Un-scaled simplified method

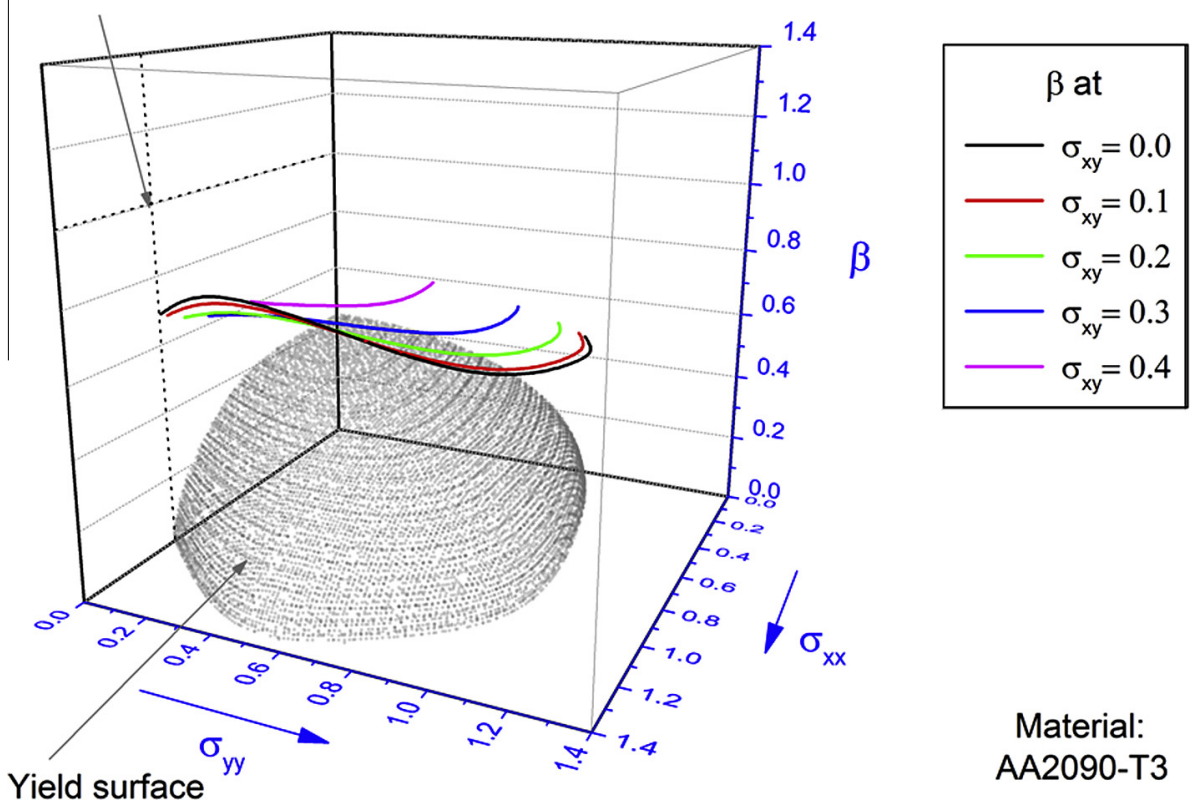

Fig. 7. Variation of $\beta$ for the un-scaled simplified non-AFR Yld2000-2d model applied to AA2090-T3.

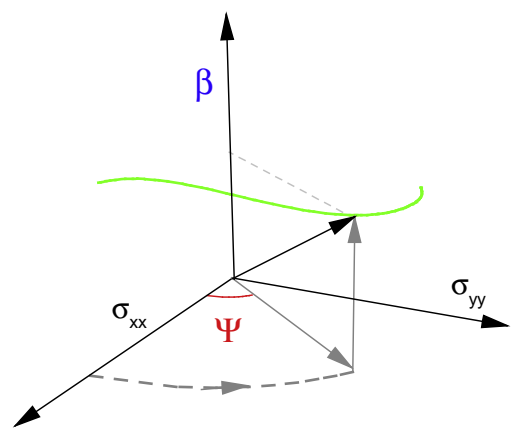

Fig. 8. Schematic of a cylindrical coordinate system.

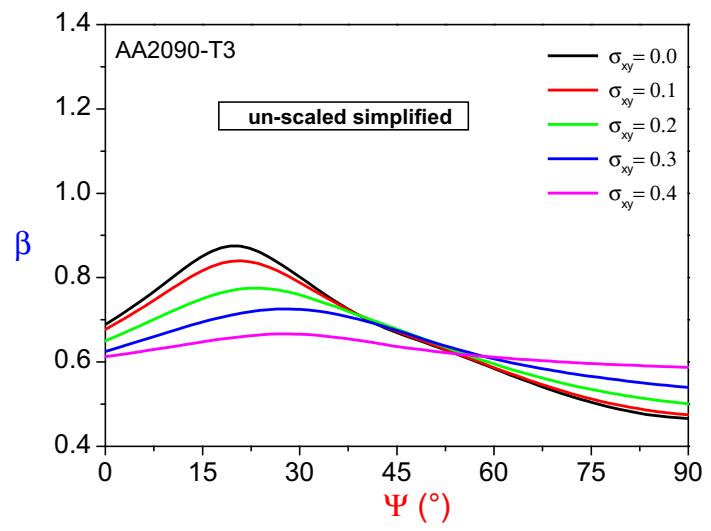

Fig. 9. Variation of $\beta$ as function of the cylindrical coordinate $\Psi$ with un-scaled simplified non-AFR Yld2000-2d model for AA2090-T3. 


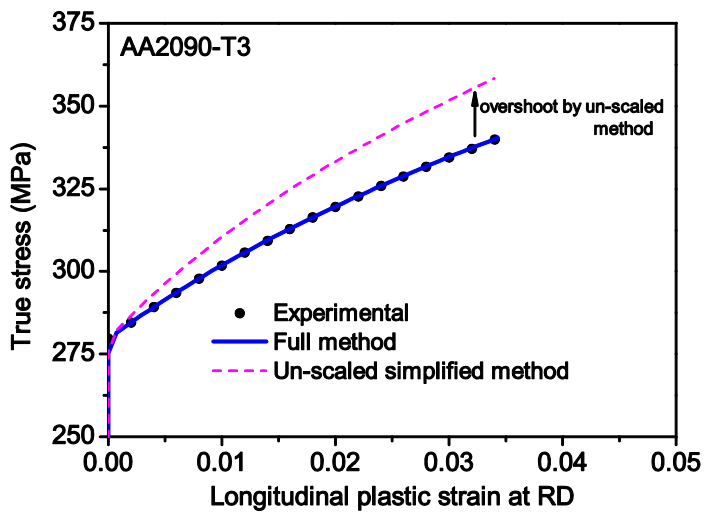

Fig. 10. Simulated stress versus longitudinal plastic strain $\left(\varepsilon_{x x}^{p}\right)$ at the rolling direction using full and un-scaled simplified methods with the non-AFR Yld2000-2d model for AA2090-T3.

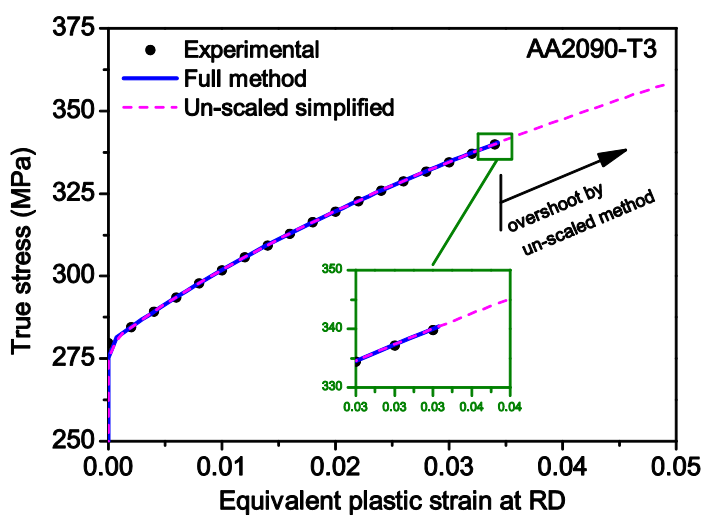

Fig. 11. Simulated stress versus equivalent plastic strain $\left(\bar{\varepsilon}^{p}\right)$ at rolling direction using full and un-scaled simplified methods with the non-AFR Yld2000-2d model for AA2090-T3.

variation of $\beta^{\text {un-scaled simplified }}$. In Fig. 9 the parameter values are plotted for different shear stress levels. This plot illustrates that the $\beta^{\text {un-scaled simplified }}$ values and thus the plastic potential $f_{p}$ are always less than one for AA2090-T3 (note that $f_{y}=1$ when yielding occurs). Consequently, substituting Eq. (24) into Eq. (28a) for a uniaxial stress state along the rolling direction, leads to

$$
d \bar{\varepsilon}^{p}>d \varepsilon_{x x}^{p}
$$

From Eq. (32) one can conclude that the un-scaled simplified approach overestimates (overshoot) the equivalent plastic strain and consequently a difference between experimental and simulated hardening curves for the rolling direction is expected (see Figs. 10 and 11).

\subsection{Scaled simplified non-AFR method}

A remedy to the observed shortcomings of the un-scaled simplified method would be enforcing a scaling constraint for which at rolling direction $d \varepsilon_{x x}^{p}=d \bar{\varepsilon}^{p}$ applies. This can be accomplished by applying a scaling of the model parameters ( $\alpha_{i=1-8}$ ) of the plastic potential function

$$
f_{p}^{(s c)}=f_{p}\left\langle k \alpha_{i=1-8}\right\rangle
$$

where

$$
\begin{aligned}
& k=\left(\frac{f_{y}}{f_{p}}\right) \mid \sigma_{x x} \rightarrow 1 \\
& \sigma_{y y} \rightarrow 0 \\
& \sigma_{x y} \rightarrow 0
\end{aligned}
$$

$f_{p}^{(s c)}$ being the scaled plastic potential function, $\alpha_{i}$ the $i$ th parameter of the plastic potential function and $\mathrm{k}$ is the scaling factor. This scaling factor is equal to 1.451 for AA2090-T3. This approach is referred to as scaled simplified method. 
Subsequently, the relation between plastic strain and equivalent plastic strain is recast to

$$
d \underset{\sim}{\varepsilon^{p}}=d \bar{\varepsilon}^{p} n_{\sim}^{(s c)}
$$

where

$$
{\underset{\sim}{\sim}}^{(s c)}=\frac{\partial f_{p}^{(s c)}}{\partial \bar{\sigma}}=k \underset{\sim}{n}
$$

therefore

$$
n_{x x}^{(s c)}=k n_{x x}
$$

The flow rule takes the following form

$$
\left(d \bar{\varepsilon}^{p}=d \lambda=\frac{d \varepsilon_{\sim}^{p}}{{\underset{\sim}{(s c)}}^{\text {scaled simplified }}}\right)^{\text {s. }}
$$

By substituting Eq. (34) into Eq. (37) and recalling Euler's theorem in Eq. (13a) for uniaxial tension along the rolling direction we can write

$$
n_{x x}^{(s c)}=1
$$

The constraint in Eq. (39) is the outcome of the scaling procedure. Finally, Eq. (35) for the uniaxial tension in the rolling direction reduces to

$$
\left(d \bar{\varepsilon}^{p}=d \varepsilon_{x x}^{p}\right)^{\text {scaled simplified }}
$$

Leading to the equality of equivalent plastic strain with longitudinal plastic strain along the rolling direction which was not possible without scaling.

Similar to Eq. (31), the $\beta^{\text {scaled simplified }}$ parameter can be obtained from

$$
\frac{\left.d \bar{\varepsilon}^{p}\right|^{\text {full method }}}{\left.d \bar{\varepsilon}^{p}\right|^{\text {scaled simplified }}}=\frac{f_{p}^{(s c)}}{f_{y}}=\beta^{\text {scaled simplified }}
$$

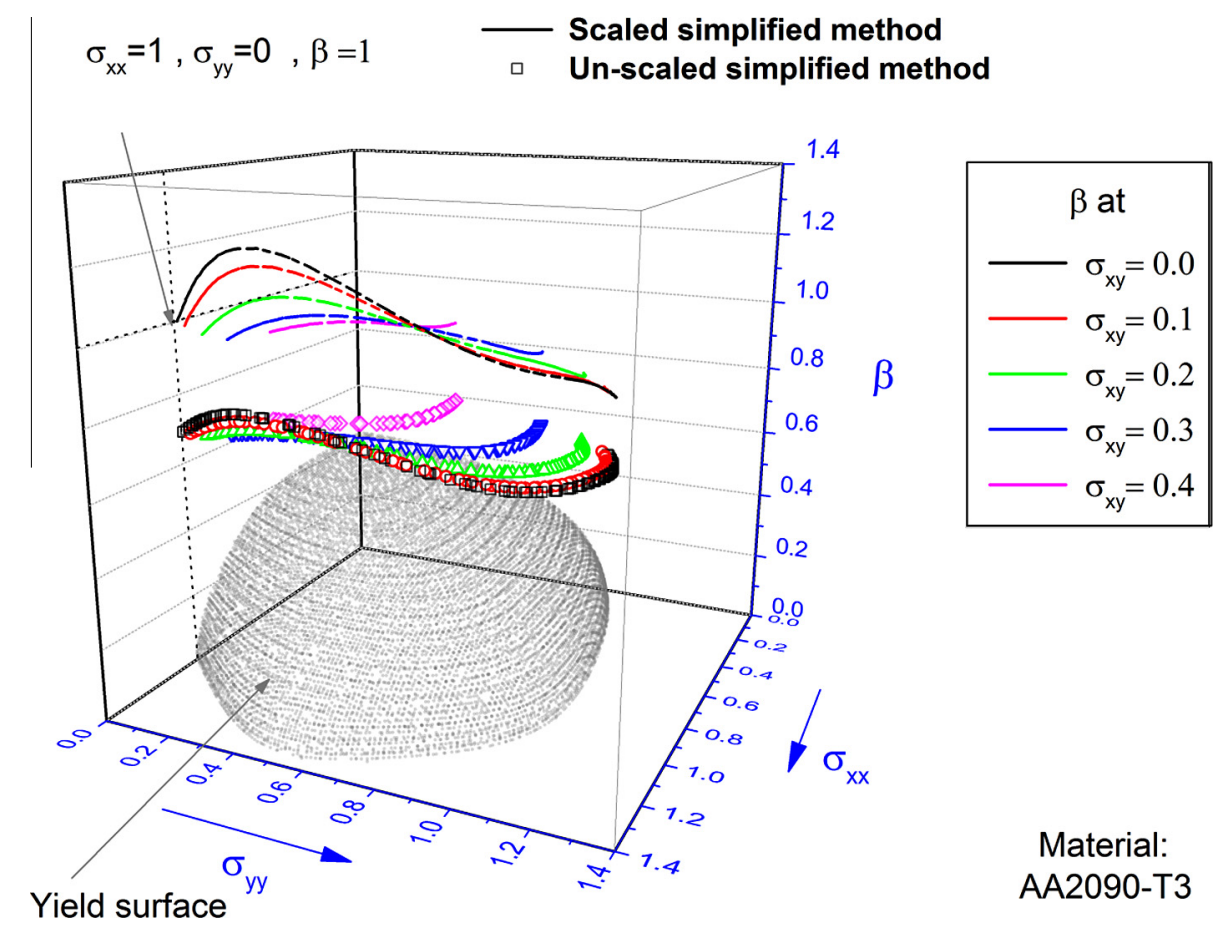

Fig. 12. Comparison of $\beta$ for scaled and un-scaled simplified non-AFR based on Yld2000-2d model with different values of shear stress for AA2090-T3. 


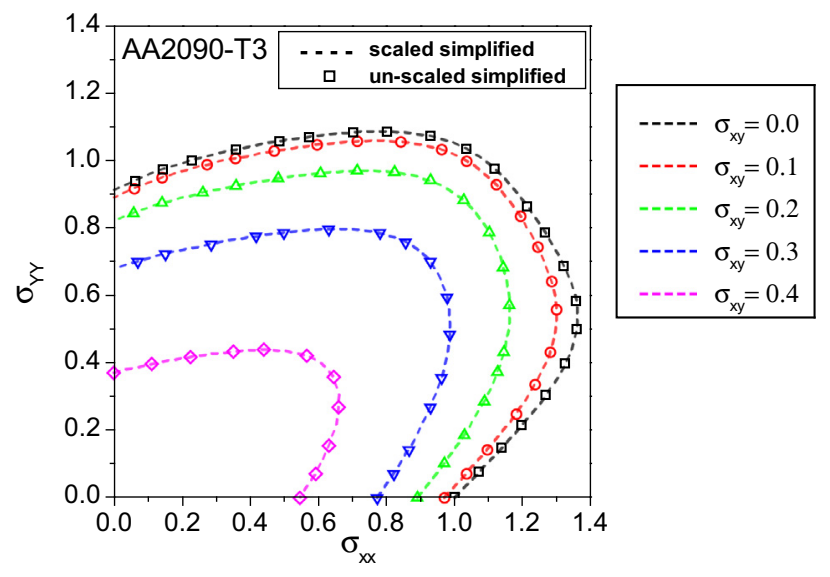

Fig. 13. Top view of Fig 12.

Fig. 12 compares the variation of $\beta^{\text {un-scaled simplified }}$ and $\beta^{\text {scaled simplified }}$ for different stress states at the first quarter of the Yld2000-2d yield surface (non-AFR version). Fig. 13 shows the top view of the same plot. In this plot it is shown that the shape of the yield function and plastic potential are not changed upon any kind of simplification.

In Fig. 14, the $\beta^{\text {un-scaled simplified }}$ and $\beta^{\text {scaled simplified }}$ values are plotted for different shear stress levels in a cylindrical coordinate system. In this plot it is shown that the $\beta^{\text {scaled simplified }}$ parameter equals to 1 along the rolling direction.

In Fig. 15, the scaling procedure is illustrated for $0^{\circ}$ and $76^{\circ}$ orientations of the in-plane stress state. In the plot, the superscripts "sc" and "un" respectively denote the scaled simplified and un-scaled simplified approaches. It can be noted that $f_{p}^{s c}$ (value of scaled plastic potential function) equals to 1 at the rolling direction.

As a consequence of the scaling method, overshooting of equivalent plastic strain and stress at rolling direction that was shown in the un-scaled method is corrected in the scaled approach (see Figs. 16 and 17).

Nonetheless, for any stress state other than the uniaxial one, the curves of $d \bar{\varepsilon}^{p}$ versus $\varepsilon^{p}$ might deviate from those obtained from the full expression. For the three methods including full, scaled simplified and un-scaled simplified the equivalent plastic strain versus uniaxial plastic strains at each $15^{\circ}$ from the rolling as well as balanced biaxial stress state are plotted in Fig. 18. As proven earlier, the equivalent and uniaxial plastic strains for scaled simplified and full expression along the rolling direction completely match with each other and very good agreement is also shown for the data at $15^{\circ}$ and $30^{\circ}$ orientations as shown in Fig. 18. However, a slight deviation is observed from $45^{\circ}$ onwards and reaches a maximum at $90^{\circ}$. Interestingly, the deviation of the un-scaled simplified method is much more pronounced. We will investigate how these deviations affect on the prediction error of the directional hardening curves compared to the experimental hardening curves.

\subsection{Metrics for error analysis}

Stoughton and Yoon (2009) conducted the error analysis based on RMS error for their anisotropic hardening model and compared the experimental hardening predictions with the several isotropic and anisotropic hardening models with RMS

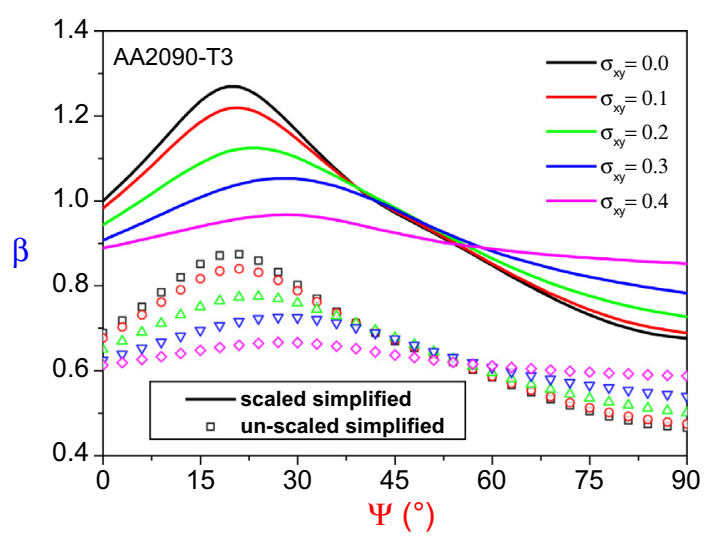

Fig. 14. Variation of $\beta$ in function of the cylindrical coordinate for scaled and un-scaled simplified non-AFR based on Yld2000-2d model for AA2090-T3. 

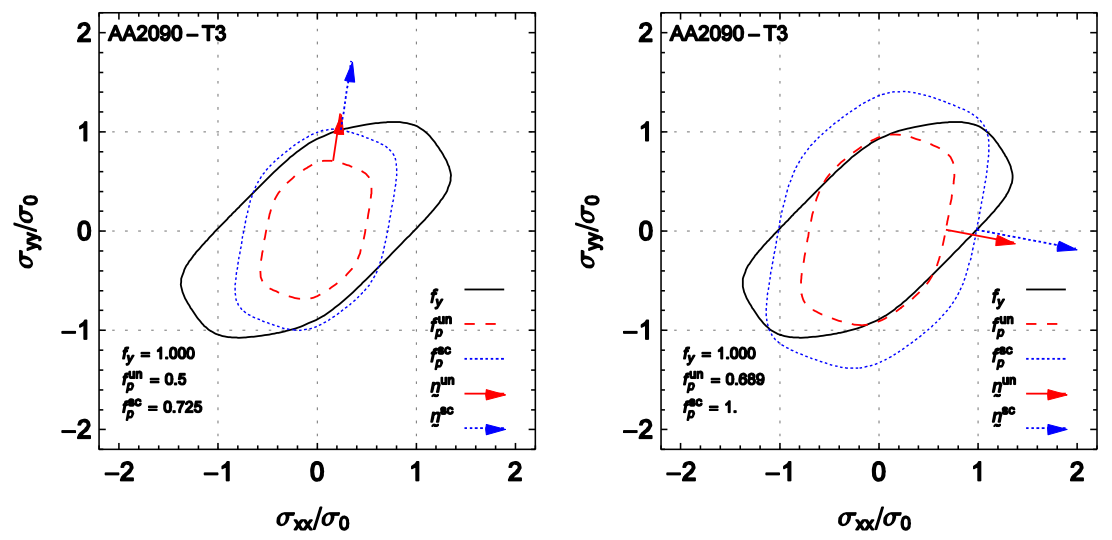

Fig. 15. Examples of scaling procedure applied to in-plane stress states at $0^{\circ}$ (right) and $76^{\circ}$ (left) orientations for AA2090-T3 and non-AFR Yld2000-2d model.

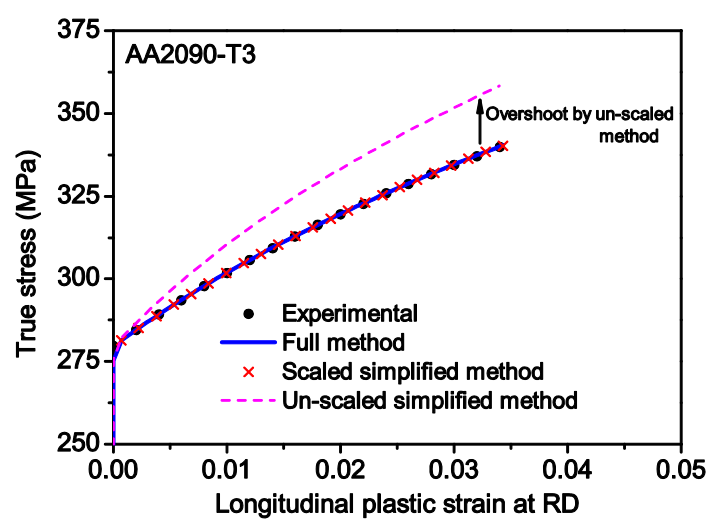

Fig. 16. Simulated stress versus longitudinal plastic strain $\left(\varepsilon_{x x}^{p}\right)$ at rolling direction using full, un-scaled simplified and scaled simplified methods for the non-AFR Yld2000-2d model applied to AA2090-T3.

error. The RMS error definitions for the three approaches used in this paper are briefly reviewed. Then, the error analysis for the full, scaled, and un-scaled approaches was conducted.

Non-associated flow rule in generic form for uniaxial tension is given as

$$
d \varepsilon_{U}^{p}=n_{U} d \lambda
$$

where $d \varepsilon_{U}^{p}$ and $n_{U}$ are respectively plastic strain and plastic flow direction for an uniaxial tension at $\theta$ degrees with respect to the rolling direction. The flow direction in any orthogonal system can be projected to the uniaxial direction denoted by the subscript $U$.

$$
n_{U}=n_{x x} \cos ^{2} \theta+n_{y y} \sin ^{2} \theta+n_{x y} \sin \theta \cos \theta
$$

Recall that in the full method, by substitution of Euler's theorem Eqs. (13a) and (13b) in Eq. (22) the plastic strain tensor is related to its conjugate equivalent value with

$$
d \varepsilon^{p}=d \bar{\varepsilon}^{p} \underset{\sim}{m}
$$

For un-scaled and scaled simplified methods, this relation is given in Eqs. (28a) and (35), respectively. Subsequently, the combinations of Eqs. (44), (28a), and (35) with Eq. (42) give

$$
\begin{aligned}
& \left(d \bar{\varepsilon}^{p}=\frac{d \varepsilon_{U}^{p}}{m_{U}}\right)^{\text {full method }} \\
& \left(d \bar{\varepsilon}^{p}=\frac{d \varepsilon_{U}^{p}}{n_{U}}\right)^{\text {un-scaled simplified }}
\end{aligned}
$$




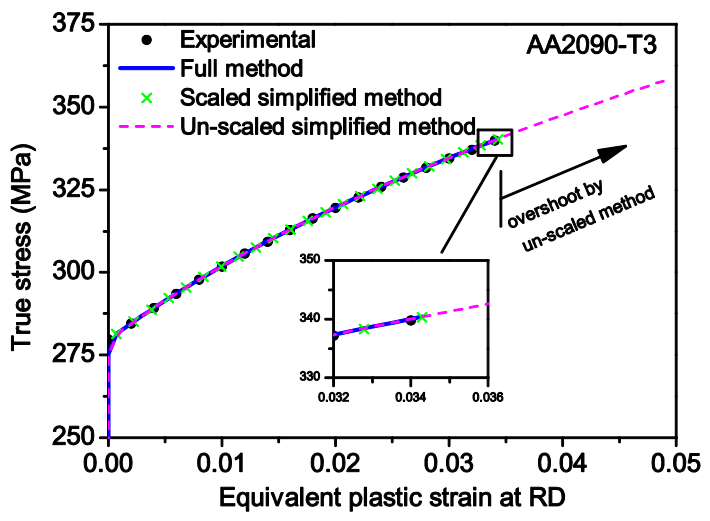

Fig. 17. Simulated stress versus equivalent plastic strain $\left(\bar{\varepsilon}^{p}\right)$ at rolling direction using full, un-scaled simplified and scaled simplified methods for the nonAFR Yld2000-2d model applied to AA2090-T3.

$$
\left(d \bar{\varepsilon}^{p}=\frac{d \varepsilon_{U}^{p}}{n_{U}^{(s)}}\right)^{\text {scaled simplified }}
$$

Next, the principle of plastic work equivalence Eq. (11) can be recast for directional uniaxial tension tests

$$
\sigma^{i s o}\left(\bar{\varepsilon}^{p}\right) d \bar{\varepsilon}^{p}=\sigma_{U}(\theta) d \varepsilon_{U}^{p}
$$

Consequently, the uniaxial stress at $\theta$ degrees with respect to the rolling direction, $\sigma_{U}(\theta)$ is determined by substitution of $d \bar{\varepsilon}^{p}$ shown from Eqs. (45)-(47) into Eq. (48). Therefore $\sigma_{U}(\theta)$

Full approach:

$$
\sigma_{U}(\theta)=\sigma^{i s o}\left(\frac{d \varepsilon_{U}^{p}}{m_{U}}\right) / m_{U}(\theta)
$$

Un-scaled simplified approach:

$$
\sigma_{U}(\theta)=\sigma^{i s o}\left(\frac{d \varepsilon_{U}^{p}}{n_{U}}\right) / m_{U}(\theta)
$$

Scaled simplified approach:

$$
\sigma_{U}(\theta)=\sigma^{i s o}\left(\frac{d \varepsilon_{U}^{p}}{n_{U}^{(s)}}\right) / m_{U}(\theta)
$$

It must be noted in above equations that $\bar{\varepsilon}^{p}=\int d \bar{\varepsilon}^{p}$ was taken into consideration.

For the balanced biaxial stress, the derivatives of plastic potential and yield function are respectively

$$
\begin{aligned}
& n_{b}=n_{x x}+n_{y y} \\
& m_{b}=m_{x x}+m_{y y}
\end{aligned}
$$

Therefore, similar to Eqs. (49)-(51) the balanced biaxial stress for the different approaches is described by:

Full approach:

$$
\sigma_{b}=\sigma^{i s o}\left(\frac{d \varepsilon_{b}^{p}}{m_{b}}\right) / m_{b}
$$

Un-scaled simplified approach:

$$
\sigma_{b}=\sigma^{i s o}\left(\frac{d \varepsilon_{U}^{p}}{n_{b}}\right) / m_{b}
$$

Scaled simplified approach:

$$
\sigma_{b}=\sigma^{i s o}\left(\frac{d \varepsilon_{U}^{p}}{n_{b}^{(s)}}\right) / m_{b}
$$

where 

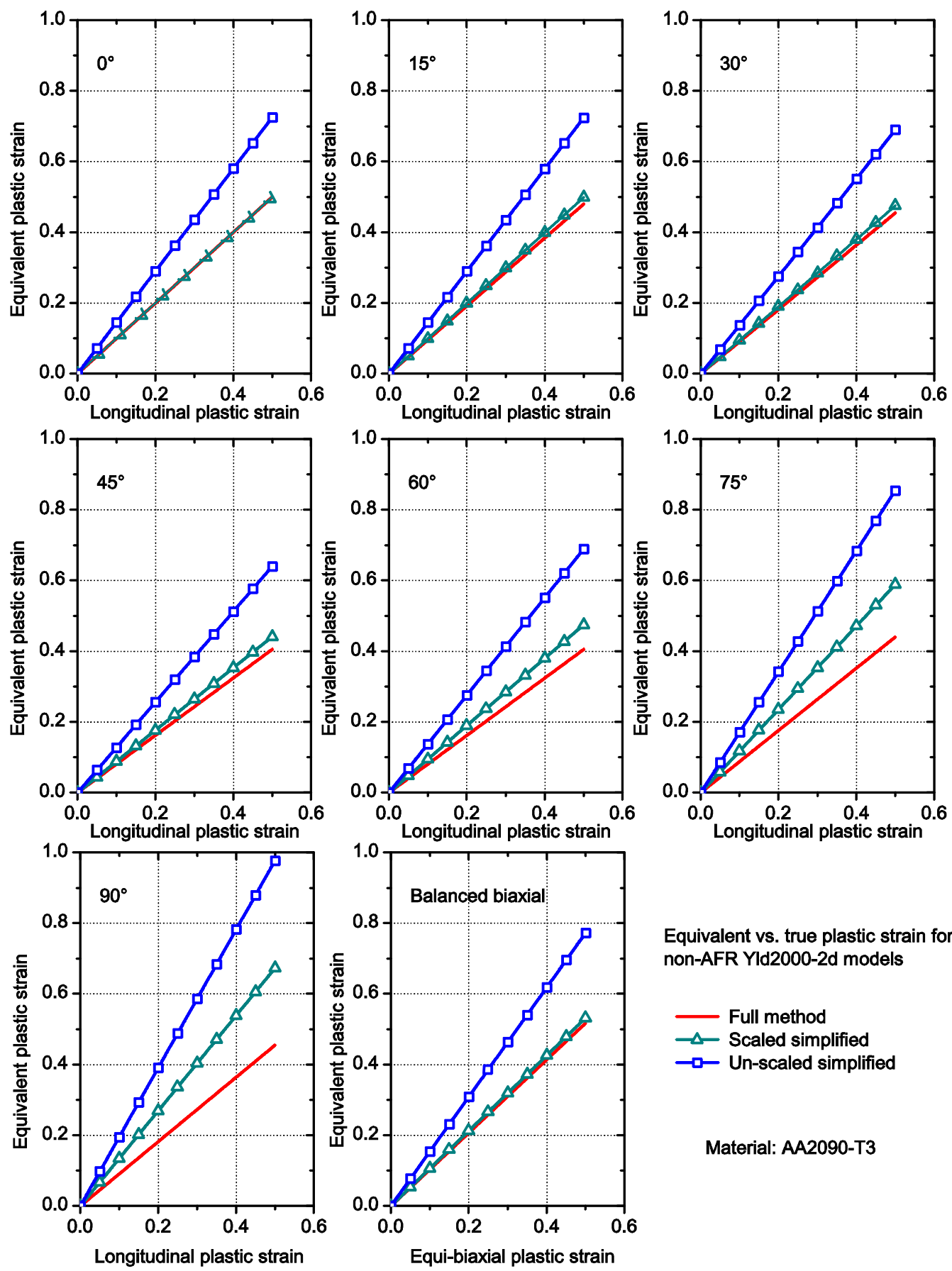

Equivalent vs. true plastic strain for non-AFR Yld2000-2d models

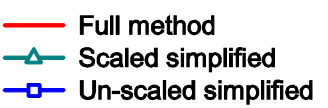

Material: AA2090-T3

Fig. 18. Equivalent versus uniaxial and equi-biaxial plastic strains at different orientations using full, scaled simplified and un-scaled simplified nonassociated flow models for AA2090-T3.

$$
n_{b}^{(s)}=k n_{b}
$$

Finally, the error between experimental and predicted stress-strain data by any of the studied models is defined as

$$
\delta_{\theta}\left(\varepsilon_{U}^{p}\right)=\frac{\sigma_{U}\left(\theta, \varepsilon_{U}^{p}\right)}{k_{\theta}\left(\varepsilon_{U}^{p}\right)}-1
$$

where $k_{\theta}\left(\varepsilon_{U}^{p}\right)$ is the experimental hardening curve at $\theta$ degrees with respect to the rolling direction and true uniaxial strain $\varepsilon_{U}^{p}$. The parameters of the experimental hardening curve are provided in Table 4 (Rousselier et al., 2009, 2010) for the different orientations. Stoughton and Yoon (2009) proposed a criterion for measuring the error at all orientations momentarily. 
Table 4

Swift hardening parameters for AA2090-T3; $\sigma=K\left(\varepsilon_{0}+\varepsilon^{p}\right)^{n}$.

\begin{tabular}{|c|c|c|c|}
\hline Orientation & $k$ & $\varepsilon_{0}$ & $n$ \\
\hline $0^{\circ}$ & 646.015 & 0.025 & 0.227 \\
\hline $15^{\circ}$ & 630.085 & 0.032 & 0.247 \\
\hline $30^{\circ}$ & 596.989 & 0.031 & 0.245 \\
\hline $45^{\circ}$ & 532.190 & 0.029 & 0.242 \\
\hline $60^{\circ}$ & 530.188 & 0.030 & 0.243 \\
\hline $75^{\circ}$ & 577.705 & 0.031 & 0.245 \\
\hline $90^{\circ}$ & 596.989 & 0.031 & 0.245 \\
\hline$b$ & 821.355 & 0.059 & 0.369 \\
\hline
\end{tabular}

The average RMS error over a uniaxial true plastic strain range of $\varepsilon_{U}^{p}$ is

$$
\Delta_{\theta}\left(\varepsilon_{U}^{p}\right)=\sqrt{\frac{1}{\varepsilon_{U}^{p}} \int_{0}^{\varepsilon_{U}^{p}}\left|\delta_{\theta}\left(\varepsilon_{U}^{p}\right)\right|^{2} d \varepsilon_{U}^{p}}
$$

The error obtained by Eq. (59) at various test conditions is shown in Fig. 19. The average RMS error for seven uniaxial directions is

$$
\Delta_{U}(\theta)=\sqrt{\frac{1}{12}\left(\begin{array}{l}
\left(\Delta_{0}\left(\varepsilon_{U}^{p}\right)\right)^{2}+2\left(\Delta_{15}\left(\varepsilon_{U}^{p}\right)\right)^{2}+2\left(\Delta_{30}\left(\varepsilon_{U}^{p}\right)\right)^{2}+ \\
2\left(\Delta_{45}\left(\varepsilon_{U}^{p}\right)\right)^{2}+2\left(\Delta_{60}\left(\varepsilon_{U}^{p}\right)\right)^{2}+2\left(\Delta_{75}\left(\varepsilon_{U}^{p}\right)\right)^{2}+\left(\Delta_{90}\left(\varepsilon_{U}^{p}\right)\right)^{2}
\end{array}\right)}
$$

In Fig. 19, it is observed that the full expression is more accurate along $75^{\circ}, 90^{\circ}$ and the scaled approach shows the better predictions along $15^{\circ}, 30^{\circ}, 45^{\circ}$ and $60^{\circ}$. A similar accuracy was obtained for the biaxial loading. While, the unscaled approach shows significant errors for the most directions.

In Fig. 20, the overall accumulated error obtained by this Eq. (60) without the biaxial loading is presented. It can be confirmed that the simplified scaled approach shows very compatible error with the full expression for the uniaxial directional loading conditions.

Taking the error of predicted balanced biaxial stress into account

$$
\delta_{b}\left(\varepsilon_{b}^{p}\right)=\frac{\sigma_{b}\left(\varepsilon_{b}^{p}\right)}{k_{b}\left(\varepsilon_{b}^{p}\right)}-1
$$

where $k_{b}\left(\varepsilon_{b}^{p}\right)$ is the experimental balanced biaxial stress-strain relation. Similarly, the RMS error over a range $\varepsilon_{b}^{p}$ is

$$
\Delta_{b}\left(\varepsilon_{b}^{p}\right)=\sqrt{\frac{1}{\varepsilon_{b}^{p}} \int_{0}^{\varepsilon_{b}^{p}}\left|\delta_{b}\left(\varepsilon_{b}^{p}\right)\right|^{2} d \varepsilon_{b}^{p}}
$$

Finally the overall model error including all considered uniaxial and balanced biaxial stress states is

$$
\Delta\left(\varepsilon^{p}\right)=\sqrt{\frac{1}{2}\left(\left(\Delta_{U}\left(\varepsilon^{p}\right)\right)^{2}+\left(\Delta_{b}\left(\varepsilon^{p}\right)\right)^{2}\right)}
$$

Consequently, Fig. 21 shows the overall model error obtained by Eq. (63). With Fig. 21, the accuracy of the simplified scaled approach is compatible with the one of the full expression. It can be concluded that the simplified scaled approach has very good balance in terms of accuracy and computational cost.

\subsection{Impact of model simplification on cup drawing simulation}

The impact of the proposed scaled simplification of the non-AFR model is evaluated by FE simulation of cylindrical cup deep drawing of AA2090-T3. The non-AFR with Yld2000-2d anisotropic function based on the full and scaled simplified expressions is studied and the results are compared with experimentally determined cup profile. User defined material subroutine is developed for ABAQUS/Standard using a fully implicit integration scheme. Material data for AA 2090-T3 and the process conditions are shown in Yoon et al. (2000).

The cup drawing process is schematized in Fig. 22 and the tool dimensions are given in Table 5. Initial sheet thickness is $1.6 \mathrm{~mm}$. In the light of orthogonal symmetry, only one quarter of the sheet was modeled. The blank was meshed using approximately 700 first order reduced integration quadrilateral shell elements with 11 Gauss integration points through the thickness. Earing profiles of AA 2090-T3 predicted from Barlat et al.'s (2005) (Yld2004-18p) and an analytical approach can be found in the works of Yoon et al. (2006, 2011), respectively. Earing simulation to predict more than four ears using Yld20000-2d under non-associated flow can be found at Yoon et al. (2007), Park and Chung (2012) and Safaei et al. (2013b).

The simulated cup profiles are plotted in Fig. 23. Because of the compatible solution demonstrated in Fig. 21, it is shown the predicted earing profiles from the simplified scaled approach and full expression are very close. The CUP times at a HP EliteBook (8530p, 2.66 GHz dual core, 4 GB RAM) are summarized for the cup drawing example; i.e., 

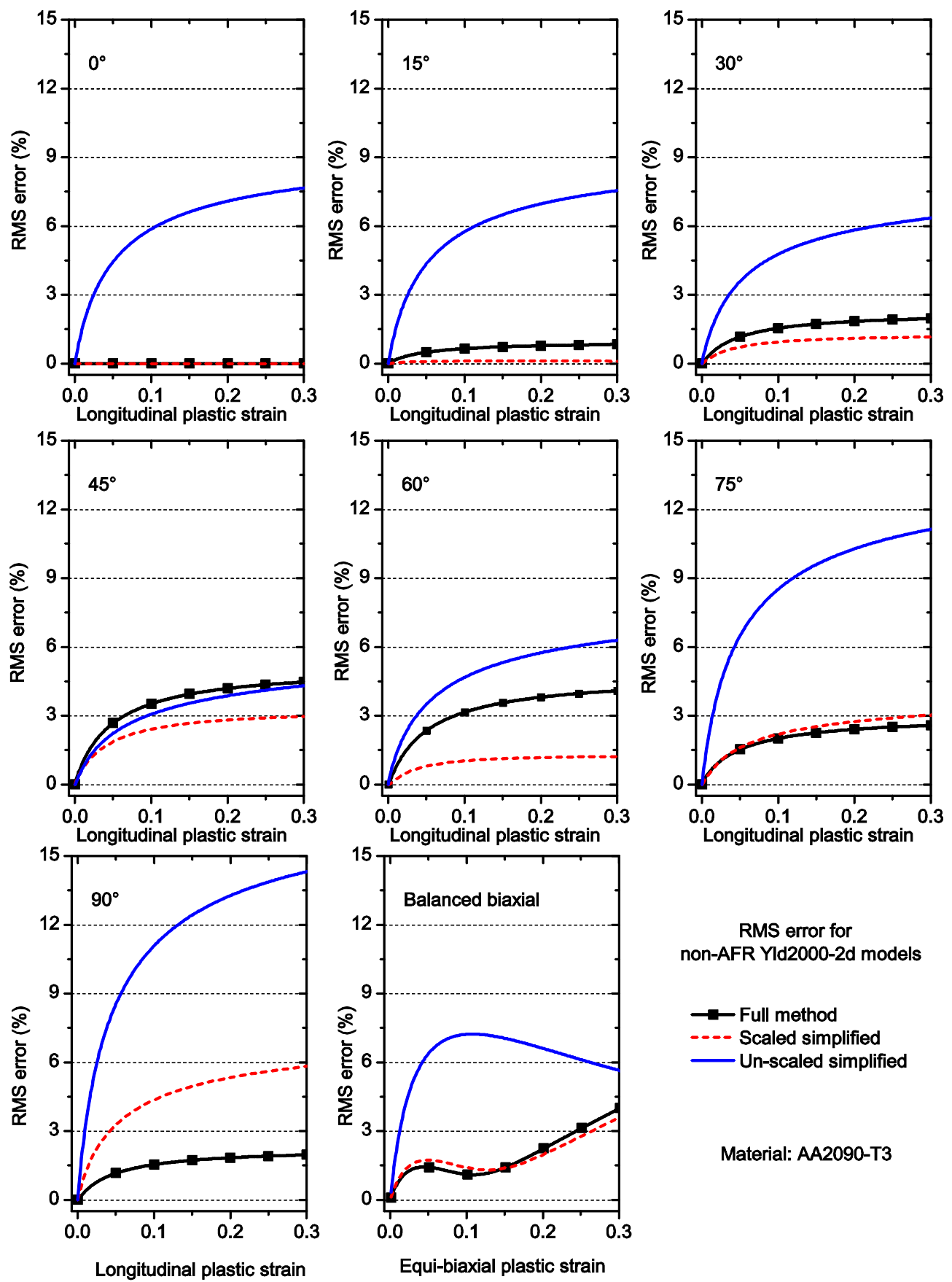

RMS error for non-AFR Yld2000-2d models

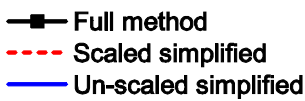

Material: AA2090-T3

Fig. 19. RMS error for prediction of stresses by full, scaled simplified and un-scaled simplified non-associated flow models for 7 uniaxial and one balanced biaxial stress conditions applied to AA2090-T3.

- Non-AFR Yld2000-2d (full method): 82 min and $39 \mathrm{~s}$

- Non-AFR Yld2000-2d (scaled simplified method): 66 min and $10 \mathrm{~s}$

Around $20 \%$ of computational time has been improved with the scaled simplified approach, which is significant saving.

As a conclusion it is proven that simplification of the non-AFR model, taking the scaling of the plastic potential function into account, leads to almost identical cup profiles as obtained using the full expression with great computational efficiency. 


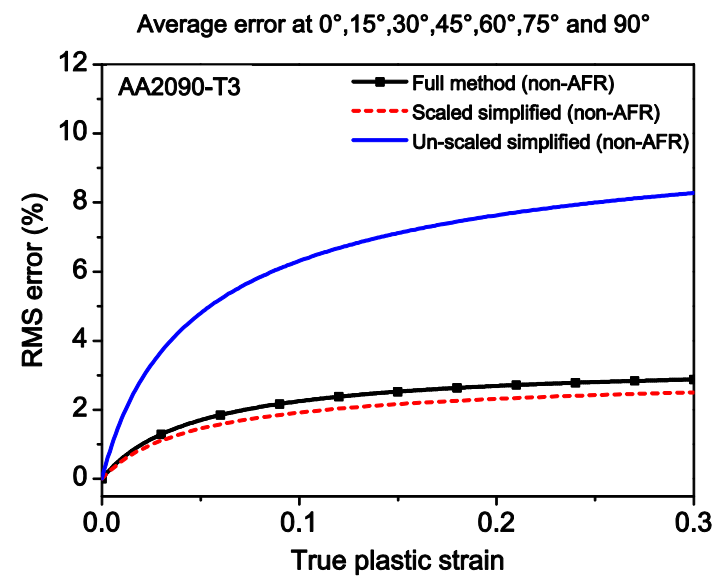

Fig. 20. Accumulated error in prediction of stress for AA2090-T3 in uniaxial loading conditions.

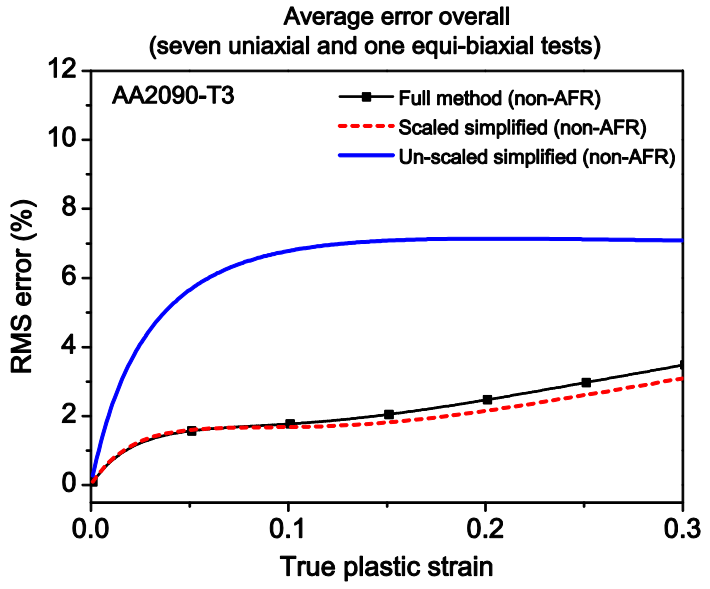

Fig. 21. Accumulated error in prediction of stress for AA2090-T3 in uniaxial and balanced biaxial loading conditions.

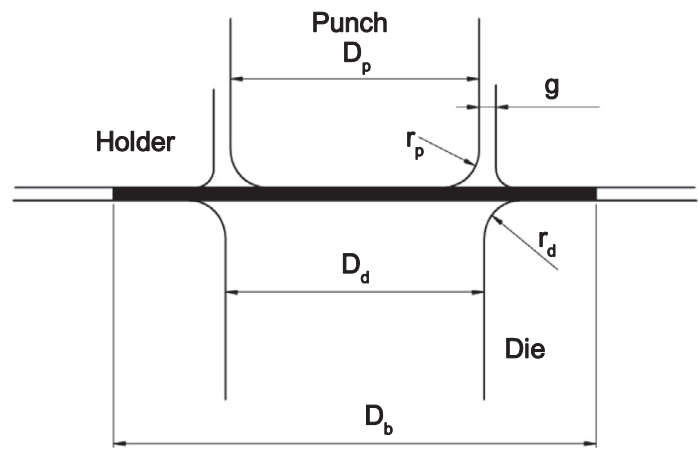

Fig. 22. Tool geometry for cylindrical cup drawing.

Table 5

Tool dimensions (unit: $\mathrm{mm}$ ).

\begin{tabular}{llllll}
\hline$D_{p}$ & $D_{d}$ & $D_{b}$ & $r_{p}$ & $r_{d}$ & \\
\hline 97.46 & 101.48 & 158.76 & 12.7 & 12.7 & 2.7 \\
\hline
\end{tabular}




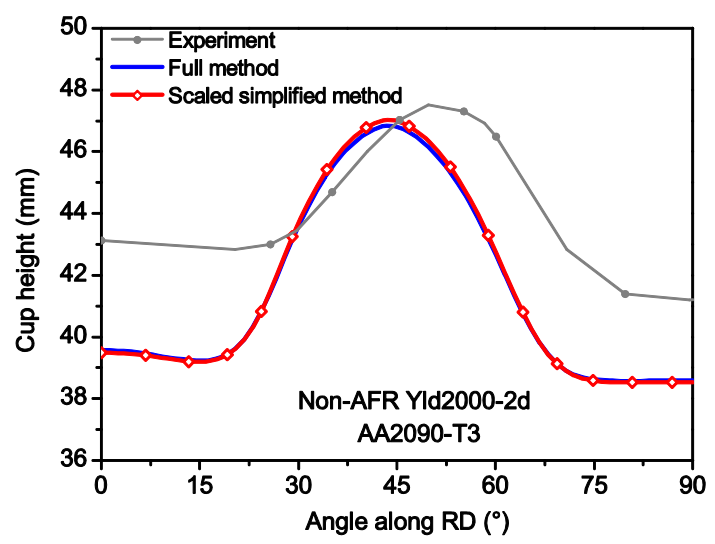

Fig. 23. Simulated cup profile using full and scaled simplified expressions of non-AFR Yld2000-2d model.

\section{Summary}

The two simplified methods for the relationship between equivalent plastic strain and compliance factor in a non-AFR model have been described. It was shown that if the non-AFR is simplified without any scaling of the plastic potential function, this results in a wrong definition of equivalent plastic strain. This inaccurate definition leads to the overestimation of Cauchy stress for AA2090-T3 due to over-prediction of equivalent plastic strain. However, it was shown that this can be corrected if the plastic potential function is scaled based on the data at uniaxial stress state. The equality of equivalent plastic strain and compliance factor can be assured resulting in a more convenient implementation scheme. FE simulation of uniaxial tensile tests at the different orientations for the non-AFR Yld2000-2d model showed that the full expression and scaled simplified approach are compatible in terms of the accumulated RMS error, although one of the two methods shows more accurate prediction along a certain direction. Finally, the cup drawing simulations of alloy AA2090-T3 using the scaled simplified approach show a very similar cup profile as compared to the one obtained from the full expression. These results prove that implementation of the scaling technique gives rise to a reliable alternative for the full expression.

\section{Acknowledgments}

Mohsen Safaei and Wim De Waele acknowledge the financial support from the Ghent University Research Fund (BOF08/ 24J/106). The authors would like to acknowledge the contribution of J. Gawad and A. Van Bael of KU Leuven for the texture measurements and the polycrystal plasticity calculations. The latter are obtained with the VEF software developed in the framework of the Knowledge Platform 'Multi-scale simulations of material forming processes' funded by the Industrial Research Fund of KU Leuven. Finally, collaboration of Kris Hertschap from ArcelorMittal Global R\&D Gent (OCAS) for provision of DC06 samples is acknowledged.

\section{References}

Barlat, F., Lege, D.J., Brem, J.C., 1991. A six-component yield function for anisotropic metals. International Journal of Plasticity 7, 693.

Barlat, F., Brem, J.C., Yoon, J.W., Chung, K., Dick, R.E., Lege, D.J., Pourgoghrat, F., Choi, S.H., Chu, E., 2003. Plane stress yield function for aluminum alloy sheets - Part 1: Theory. International Journal of Plasticity 19, 1297-1319.

Barlat, F., Aretz, H., Yoon, J.W., Karabin, M.E., Brem, J.C., Dick, R.E., 2005. Linear transformation-based anisotropic yield functions. International Journal of Plasticity $21,1009-1039$.

Barlat, F., Yoon, J.W., Cazacu, O., 2007. On linear transformations of stress tensors for the description of plastic anisotropy. International Journal of Plasticity $23,876-896$.

Barlat, F., Gracio, J.J., Lee, M.G., Rauch, E.F., Vincze, G., 2011. An alternative to kinematic hardening in classical plasticity. International Journal of Plasticity 27, 1309-1327.

Bishop, J.F.W., Hill, R., 1951. XLVI. A theory of the plastic distortion of a polycrystalline aggregate under combined stresses. Philosophical Magazine Series 7 $42,414-427$.

Bron, F., Besson, J., 2004. A yield function for anisotropic materials - application to aluminum alloys. International Journal of Plasticity 20, 937-963.

Cazacu, O., Barlat, F., 2002. A new anisotropic yield criterion for aluminum alloys. Advanced Materials Forum I 230-2, 537-540.

Cazacu, O., Barlat, F., 2004. A criterion for description of anisotropy and yield differential effects in pressure-insensitive metals. International Journal of Plasticity 20, 2027-2045.

Cazacu, O., Barlat, F., Nixon, M.E., 2004. New anisotropic constitutive models for HCP sheet forming simulations. In: Ghosh, S., Castro, J.M., Lee, J.K. (Eds.), Materials Processing and Design: Modeling, Simulation and Applications, Pts 1 and 2, American Institute of Physics. Melville, pp. 1046-1051.

Cazacu, O., Plunkett, B., Barlat, F., 2006. Orthotropic yield criterion for hexagonal closed packed metals. International Journal of Plasticity 22, 1171-1194.

Chung, K., Lee, S.Y., Barlat, F., Keum, Y.T., Park, J.M., 1996. Finite element simulation of sheet forming based on a planar anisotropic strain-rate potential. International Journal of Plasticity 12, 93-115.

Cleja-Tigoiu, S., Iancu, L., 2013. Orientational anisotropy and strength-differential effect in orthotropic elasto-plastic materials. International Journal of Plasticity $47,80-110$. 
Cvitanic, V., Vlak, F., Lozina, Z., 2008. A finite element formulation based on non-associated plasticity for sheet metal forming. International Journal of Plasticity 24, 646-687.

Gao, X., Zhang, T., Zhou, J., Graham, S.M., Hayden, M., Roe, C., 2011. On stress-state dependent plasticity modeling: significance of the hydrostatic stress, the third invariant of stress deviator and the non-associated flow rule. International Journal of Plasticity 27, $217-231$.

Gawad, J., Van Bael, A., Eyckens, P., Samaey, G., Van Houtte, P., Roose, D., 2013. Hierarchical multi-scale modeling of texture induced plastic anisotropy in sheet forming. Computational Materials Science 66, 65-83.

Hecker, S.S., 1976. Experimental studies of yield phenomena in biaxially loaded metals. In: Stricklin, A., Saczalski, K.C. (Eds.), Constitutive Modelling in Viscoplasticity. ASME, New York, pp. 1-33.

Hill, R., 1948. A theory of the yielding and plastic flow of anisotropic metals. Proceedings of the Royal Society of London Series A. Mathematical and Physical Sciences 193, 281-297.

Hu, W., 2007. Constitutive modeling of orthotropic sheet metals by presenting hardening-induced anisotropy. International Journal of Plasticity 23, 620639.

Karafillis, A.P., Boyce, M.C., 1993. A general anisotropic yield criterion using bounds and a transformation weighting tensor. Journal of the Mechanics and Physics of Solids 41, 1859-1886.

Park, T., Chung, K., 2012. Non-associated flow rule with symmetric stiffness modulus for isotropic-kinematic hardening and its application for earing in circular cup drawing. International Journal of Solids and Structures 49, 3582-3593.

Rousselier, G., Barlat, F., Yoon, J.W., 2009. A novel approach for anisotropic hardening modeling. Part I: Theory and its application to finite element analysis of deep drawing. International Journal of Plasticity 25, 2383-2409.

Rousselier, G., Barlat, F., Yoon, J.W., 2010. A novel approach for anisotropic hardening modeling. Part II: Anisotropic hardening in proportional and nonproportional loadings, application to initially isotropic material. International Journal of Plasticity 26, 1029-1049.

Safaei, M., Lee, M.G., Zang, S.L., De Waele, W., 2013a. An evolutionary anisotropic model for sheet metals based on non-associated flow rule approach. Computational Materials Science. http://dx.doi.org/10.1016/j.commatsci.2013.05.035.

Safaei, M., Zang, S.-1., Lee, M.-G., De Waele, W., 2013b. Evaluation of anisotropic constitutive models: Mixed anisotropic hardening and non-associated flow rule approach. International Journal of Mechanical Sciences 73, 53-68.

Simo, J.C., Hughes, T.J.R., 1998. Computational inelasticity. Interdisciplinary Applied Mathematics. Springer, New York, ISBN 0387975209.

Stoughton, T.B., 2002. A non-associated flow rule for sheet metal forming. International Journal of Plasticity 18, $687-714$.

Stoughton, T.B., Yoon, J.W., 2004. A pressure-sensitive yield criterion under a non-associated flow rule for sheet metal forming. International Journal of Plasticity 20, 705-731.

Stoughton, T.B., Yoon, J.W., 2006. Review of Drucker's postulate and the issue of plastic stability in metal forming. International Journal of Plasticity 22, 391433.

Stoughton, T.B., Yoon, J.W., 2008. On the existence of indeterminate solutions to the equations of motion under non-associated flow. International Journal of Plasticity 4, 583-613.

Stoughton, T.B., Yoon, J.W., 2009. Anisotropic hardening and non-associated flow in proportional loading of sheet metals. International Journal of Plasticity $25,1777-1817$.

Taherizadeh, A., Green, D.E., Ghaei, A., Yoon, J.W., 2010. A non-associated constitutive model with mixed iso-kinematic hardening for finite element simulation of sheet metal forming. International Journal of Plasticity 26, 288-309.

Taherizadeh, A., Green, D.E., Yoon, J.W., 2011. Evaluation of advanced anisotropic models with mixed hardening for general associated and non-associated flow metal plasticity. International Journal of Plasticity 27, 1781-1802.

Van Houtte, P., 1995. The MTM-FHM Software System Version 2, Department of Metallurgy and Materials Engineering, KU Leuven.

Van Houtte, P., Li, S., Seefeldt, M., Delannay, L., 2005. Deformation texture prediction: from the Taylor model to the advanced Lamel model. International Journal of Plasticity 21, 589-624.

Van Houtte, P., Gawad, J., Eyckens, P., Bael, B., Samaey, G., Roose, D., 2011. A full-field strategy to take texture-induced anisotropy into account during FE simulations of metal forming processes. JOM 63, 37-43.

Vegter, H., van den Boogaard, A.H., 2006. A plane stress yield function for anisotropic sheet material by interpolation of biaxial stress states. International Journal of Plasticity 22, 557-580.

Yoon, J.W., Yang, D.Y., Chung, K., 1999. Elasto-plastic finite element method based on incremental deformation theory and continuum based shell elements for planar anisotropic sheet materials. Computer Methods in Applied Mechanics 174, 23-56.

Yoon, J.W., Barlat, F., Chung, K., Pourboghrat, F., Yang, D.Y., 2000. Earing predictions based on asymmetric nonquadratic yield function. International Journa of Plasticity 16, 1075-1104.

Yoon, J.W., Barlat, F., Dick, R.E., Chung, K., Kang, T.J., 2004. Plane stress yield function for aluminum alloy sheets - Part II: FE formulation and its implementation. International Journal of Plasticity 20, 495-522.

Yoon, J.W., Barlat, F., Dick, R.E., Karabin, M.E., 2006. Prediction of six or eight ears in a drawn cup based on a new anisotropic yield function. International Journal of Plasticity 22, 174-193.

Yoon, J.W., Stoughton, T.B., Dick, R.E., 2007. Earing prediction in cup drawing based on non-associated flow rule. In: CeasarDeSa, J.M.A., Santos, A.D. (Eds.), NUMIFORM '07: Materials Processing and Design: Modeling, Simulation and Applications, Pts I and II, American Institute of Physics. Melville, pp. 685690.

Yoon, J.W., Barlat, F., Dick, R.E., 2011. A new analytical theory for earing generated from anisotropic plasticity. International Journal of Plasticity 27, 11651184. 